\title{
Concurrence Imparfaite, Variabilité du Taux de Marge et Fluctuations Endogènes
}

\author{
Thomas Seegmuller*
}

26 avril 2002

*BETA-Theme, Université Louis Pasteur, 61, avenue de la Forêt-Noire, 67085 Strasbourg Cedex, France, Tel: (33) 3902420 98, Fax: (33) 3902420 71, e-mail: seegmu@cournot.u-strasbg.fr. Je tiens à remercier Antoine d'Autume, Francesco De Palma, Rodolphe Dos Santos Ferreira, Teresa Lloyd-Braga, Isabelle Maret, Hubert Stahn, Alain Venditti et un rapporteur anonyme pour leurs commentaires et leurs suggestions. Les erreurs et omissions qui pourraient subsister sont de la seule responsabilité de l'auteur. 


\title{
Résumé
}

Nous étudions l'émergence de fluctuations endogènes dans un modèle à générations imbriquées dans lequel les producteurs se livrent une concurrence à la Cournot avec libre entrée. L'économie est caractérisée par un taux de marge variable et des rendements croissants. Nous analysons l'influence de la variabilité du taux de marge et du niveau des rendements croissants sur l'indétermination locale de l'équilibre stationnaire et l'existence de cycles endogènes. Nous montrons que des fluctuations endogènes déterministes peuvent émerger si les rendements sont faiblement croissants et sont proches des rendements constants. En revanche, lorsque les rendements sont constants et le taux de marge disparaît, l'équilibre peut être indéterminé, mais des cycles déterministes ne peuvent pas apparaître. La concurrence imparfaite et la variabilité du taux de marge favorisent donc l'émergence de fluctuations endogènes.

Classification JEL: D43, E32.

Mots-clé: Fluctuations endogènes, concurrence imparfaite, variabilité du taux de marge.

\begin{abstract}
We study the emergence of endogenous fluctuations in an overlapping generations model where there is Cournot competition with free entry. The mark-up is variable and the returns to scale are increasing. We analyze the role of mark-up variability and increasing returns on the local indeterminacy and on the occurrence of endogenous cycles. We show that endogenous deterministic fluctuations can emerge if the increasing returns are small and closed to constant returns. However, when the returns are constant and the mark-up disappears, the steady state can be locally indeterminate, but endogenous deterministic cycles cannot emerge. So, imperfect competition and mark-up variability promote the emergence of endogenous fluctuations.
\end{abstract}




\section{Introduction}

Dans les vingt dernières années, de nombreux travaux ont cherché à établir les conditions qui favorisent l'émergence de fluctuations endogènes. La plupart de ces travaux se placent dans un cadre parfaitement concurrentiel. Ils montrent entre autres que la dominance de l'effet revenu sur l'effet de substitution intertemporelle (Benhabib et Day (1982), Grandmont (1985)) ou encore la complémentarité entre le travail et le capital (Grandmont, Pintus, et de Vilder (1998), Reichlin (1986), de Vilder (1996), Woodford (1986)) permettent l'existence de fluctuations endogènes. ${ }^{0}$ Ces conditions apparaissent cependant comme étant restrictives. Aussi certains auteurs ont-ils introduit de la concurrence imparfaite sur le marché du bien final, pour étudier son influence sur l'émergence de telles fluctuations. La concurrence imparfaite est, en effet, compatible avec des rendements d'échelle croissants et permet l'existence d'un taux de marge positif et éventuellement variable. Dans de tels modèles, des rendements suffisamment croissants sont souvent requis pour que des fluctuations endogènes apparaissent (Benhabib et Farmer (1994), Rivard (1994)). Or, si des travaux empiriques ont fourni un support empirique à des rendements fortement croissants (Caballero et Lyons (1992)), des résultats plus récents montrent que si les rendements sont croissants, il le sont faiblement et sont proches des rendements constants (Basu et Fernald (1995), Basu et Fernald (1997), Burnside (1996)).

Dans cet article, nous étudions l'émergence de fluctuations endogènes dans un modèle à générations imbriquées dans lequel la concurrence est imparfaite. L'économie est caractérisée par un taux de marge variable et des rendements croissants. Nous analysons l'influence de la variabilité du taux de marge et du niveau des rendements croissants sur l'indétermination locale de l'équilibre stationnaire et l'existence de cycles endogènes. Nous montrons que des fluctuations endogènes déterministes peuvent émerger même si les rendements sont faiblement croissants et sont proches des rendements constants.

Certains travaux ont déjà montré que la concurrence imparfaite et la variabilité du taux de marge favorisent l'émergence de fluctuations endogènes. Woodford (1991) montre l'existence d'équilibres à taches solaires dans un modèle oligopolistique dans lequel les producteurs ont une demande coudée. Gali (1994) étudie un modèle de concurrence monopolistique. Il montre que l'équilibre stationnaire est localement indéterminé à cause de la variabilité du taux de marge, qui est due à la différence des élasticités de la demande de biens de consommation et de biens d'investissement. Le taux de marge est

1. Pour une revue de la littérature, on peut se référer à Boldrin et Woodford (1990) ou à Guesnerie et Woodford (1992). 
alors une fonction de la part de l'investissement dans la production. L'article de d'Aspremont, Dos Santos Ferreira, et Gérard-Varet (1995) analyse un modèle de concurrence monopolistique cournotienne dans lequel l'élasticité de la demande dépend des prix. Les auteurs montrent que la variabilité du taux de marge est un déterminant de l'émergence de cycles endogènes de période deux.

Dans cet article, la variabilité du taux de marge est due à celle du nombre de producteurs (Portier (1995), Weder (2000)). Une manière de modéliser ce phénomène est de supposer que les producteurs se livrent une concurrence à la Cournot avec libre entrée sur le marché du bien final et qu'il existe des rendements croissants dus à l'existence d'un coût fixe. L'économie est alors caractérisée par la pro-cyclicité de l'entrée des firmes et la contra-cyclicité du taux de marge. Ces deux propriétés dynamiques correspondent à une observation empirique (Bils (1987), Martins et Scarpetta (1999), Rotemberg et Woodford (1991)). Portier (1995) en montre, en particulier, la validité empirique à partir de données françaises.

Nous supposons, par ailleurs, que les consommateurs ont une désutilité du travail linéaire. Depuis l'article de Hansen (1985), cette hypothèse a été fréquemment utilisée dans les modèles macroéconomiques dynamiques (Cook (2001), Coimbra (1999), Coimbra, Lloyd-Braga, et Modesto (2000), d'Aspremont, Dos Santos Ferreira, et Gérard-Varet (1995)). Nous considérons ainsi la situation où l'offre de travail est infiniment élastique.

L'étude de la dynamique locale nous permet d'analyser l'émergence de fluctuations endogènes. Nous utilisons la méthode géométrique développée par Grandmont, Pintus, et de Vilder (1998), qui s'applique aux systèmes dynamiques non-linéaires discrets à deux dimensions. Nous montrons que des fluctuations endogènes déterministes et stochastiques peuvent émerger lorsque le taux de marge est positif et variable. En effet, l'équilibre stationnaire peut être localement indéterminé et des trajectoires déterministes peuvent apparaître autour de l'équilibre stationnaire, à travers l'émergence d'une bifurcation Hopf ou flip. Il existe de telles fluctuations dès lors que le taux de marge est positif et variable et les rendements croissants. Par ailleurs, la demande de travail n'a pas besoin d'être croissante et d'avoir une pente supérieure à celle de l'offre de travail pour qu'il existe un cycle déterministe. Dans le cas limite où les rendements sont constants, un nombre infini de firmes entrent, le taux de marge disparaît et la concurrence est parfaite. Alors, l'équilibre stationnaire peut être localement indéterminé, mais des fluctuations endogènes déterministes ne peuvent pas apparaître. Aussi la présence de concurrence imparfaite et la variabilité du taux de marge favorisent-elles l'émergence de fluctuations endogènes. Si l'existence de cycles déterministes requiert des rendements croissants, ceux-ci n'ont pas besoin d'être impor- 
tants, ce qui semble en accord avec les travaux empiriques récents mentionnés au début de cette introduction.

Plusieurs autres travaux ont analysé les effets liés à la variabilité du taux de marge due au nombre variable de producteurs sur l'émergence de fluctuations endogènes. Parmi ceux-ci, Jacobsen (1998) considère un modèle de concurrence monopolistique dans lequel l'élasticité de substitution entre les différentes variétés de bien est variable. Cependant, contrairement au modèle présenté dans cet article, il n'y a pas de capital productif et la dynamique est donc uni-dimensionnelle. Kuhry (2001) propose une analyse proche de la nôtre, mais se place dans un modèle à la Woodford (1986), tandis que nous considérons un modèle à générations imbriquées. Dos Santos Ferreira et Lloyd-Braga (2000) et Seegmuller (2001) étudient, quant à eux, les effets de la substitution entre le capital et le travail sur l'émergence de fluctuations endogènes, alors que nous analysons ici le rôle des rendements croissants et du niveau du taux de marge.

Cet article peut également être relié au travail récent de Cazzavillan (2001) qui montre que des fluctuations endogènes peuvent apparaître dans un modèle à générations imbriquées lorsque les rendements sont faiblement croissants, si l'élasticité de l'offre de travail est suffisamment importante. Cet auteur analyse un modèle de concurrence parfaite où les rendements croissants sont dus à des externalités, alors que nous considérons des rendements croissants internes, la concurrence imparfaite et le taux de marge variable. Par ailleurs, si nous nous restreignons à la situation où l'offre de travail est infiniment élastique, nous supposons, contrairement à son analyse, que les consommateurs consomment aux deux périodes de leur vie.

La suite de ce travail s'organise de la façon suivante. Nous exposons le modèle et définissons l'équilibre intertemporel dans la section 2. Dans la section 3, nous étudions la dynamique locale dans le voisinage de l'équilibre stationnaire, avant de conclure dans la section 4 .

\section{Le Modèle}

Dans cette section, nous présentons le modèle à générations imbriquées dans lequel nous menons l'analyse. Nous étudions d'abord le comportement des consommateurs, puis celui des firmes, et définissons finalement l'équilibre intertemporel. 


\subsection{Les Consommateurs}

Les consommateurs sont en générations imbriquées et ont des prévisions parfaites. A chaque période $t=1,2, \ldots, \infty$, un continu de consommateurs identiques, de masse unitaire, naît et vit deux périodes. C'est pourquoi à chaque période, une génération de jeunes et une génération de vieux coexistent et la population est constante. Chaque jeune consommateur offre du travail aux firmes et est rémunéré pour cela au taux de salaire concurrentiel. Le travail est choisi comme numéraire. Il décide également de sa consommation en bien final et du montant de son épargne sous forme de capital. ${ }^{1}$ Cette épargne est rémunérée au prix de location du capital $q_{t}$ et les consommateurs l'utilisent pour consommer le bien final lorsqu'ils sont vieux. A la première période, une génération de vieux vit une seule période et possède le stock de capital $K_{0}$ comme dotation initiale.

Un jeune consommateur doit résoudre:

$$
\max C_{1 t}^{\alpha} C_{2 t+1}^{1-\alpha}-v L_{t}
$$

sous les contraintes :

$$
\begin{gathered}
p_{t} C_{1 t}+p_{t} K_{t}=L_{t} \\
p_{t+1} C_{2 t+1}=q_{t+1} K_{t}
\end{gathered}
$$

où $C_{1 t}$ est la consommation en bien final d'un jeune en t, $C_{2 t+1}$ sa consommation quand il est vieux, $K_{t}$ son épargne en capital et $L_{t} \in\left[0, L^{*}\right]$ son offre de travail, avec $L^{*}>1$. Concernant les paramètres, $\alpha \in(0,1)$ est la propension marginale à consommer des jeunes et $v>0$ la désutilité marginale du travail.

Etant donné le revenu, nous déduisons:

$$
\begin{gathered}
C_{1 t}=\alpha \frac{1}{p_{t}} L_{t} \\
C_{2 t+1}=r_{t+1}(1-\alpha) \frac{1}{p_{t}} L_{t} \\
K_{t}=(1-\alpha) \frac{1}{p_{t}} L_{t}
\end{gathered}
$$

où $r_{t+1}=\frac{q_{t+1}}{p_{t+1}}$ est le facteur d'intérêt réel. En substituant (4) et (5) dans l'expression (1), nous obtenons l'utilité indirecte d'un jeune consommateur:

2. La dépréciation du capital est totale au cours d'une période. 


$$
V_{t} \equiv \alpha^{\alpha}(1-\alpha)^{1-\alpha} r_{t+1}^{1-\alpha} \frac{1}{p_{t}} L_{t}-v L_{t}
$$

L'offre de travail du consommateur est égale à sa dotation en travail $L^{*}>1$ si $V_{t}>0$, est égale à 0 si $V_{t}<0$ et est égale à $L_{t} \in\left[0, L^{*}\right]$ si $V_{t}=0$. Nous considérons la situation où $V_{t}=0$, c'est-à-dire :

$$
\alpha^{\alpha}(1-\alpha)^{1-\alpha} r_{t+1}^{1-\alpha} \frac{1}{p_{t}}=v
$$

L'offre de travail a donc une élasticité infinie. ${ }^{2}$

\subsection{Les Firmes}

Concernant la production, $n_{t}\left(n_{t} \geq 2\right)$ firmes produisent un bien homogène à partir de deux facteurs de production, le capital et le travail, avec la technologie à rendements d'échelle croissants :

$$
y_{t}=A \max \left\{0 ; k_{t-1}^{\beta} l_{t}^{1-\beta}-f\right\}
$$

où $y_{t}$ est la quantité produite par une firme, $k_{t-1}$ le stock de capital et $l_{t}$ le travail qu'elle utilise pour la production. Concernant les paramètres, $A>0$, $\beta \in(0,1)$ et $f \in(0,1 / 4)$ un coût fixe. ${ }^{3}$ De manière alternative, cette fonction de production s'écrit:

$$
y_{t}=A \max \left\{0 ; a_{t}^{\beta} l_{t}-f\right\}
$$

où $a_{t}=\frac{k_{t-1}}{l_{t}}$ est le capital par tête.

Les firmes se comportent de manière concurrentielle sur les marchés du travail et du capital. Par contre, sur le marché du bien final, elles se livrent une concurrence à la Cournot. L'existence de concurrence imparfaite sur le marché du bien final est compatible avec l'existence de rendements d'échelle croissants. Les producteurs anticipent parfaitement la demande en bien final $D_{t}\left(p_{t}\right)$. Celle-ci est composée de la consommation des jeunes, de celle des vieux et de l'investissement privé. Plus formellement, elle s'écrit:

$$
D_{t}\left(p_{t}\right)=C_{1 t}+C_{2 t}+K_{t}=\frac{L_{t}+q_{t} K_{t-1}}{p_{t}}
$$

3. Nous n'étudions pas le cas où $V_{t}>0$ et $L_{t}=L^{*}$, car la dynamique est alors unidimensionnelle et concerne uniquement le stock de capital.

4. L'importance de l'hypothèse $f<\frac{1}{4}$ apparaîtra clairement lorsque nous étudierons la dynamique locale. 
Chaque firme choisit une combinaison optimale des facteurs de production de façon à maximiser son profit :

$$
D_{t}^{-1}\left(y_{t}+\bar{Y}_{t}\right) y_{t}-q_{t} k_{t-1}-l_{t}
$$

où $D_{t}^{-1}\left(y_{t}+\bar{Y}_{t}\right)$ est la demande inverse déduite de l'équation (11) et $\bar{Y}_{t}$ est la quantité de bien final offerte par les concurrents. Comme $\frac{D_{t}^{\prime}\left(p_{t}\right) p_{t}}{D_{t}\left(p_{t}\right)}=-1$, les conditions du premier ordre de la firme s'écrivent ${ }^{4}$ :

$$
\begin{gathered}
p_{t}\left[1-\frac{y_{t}}{y_{t}+\bar{Y}_{t}}\right] \beta A a_{t}^{\beta-1}=q_{t} \\
p_{t}\left[1-\frac{y_{t}}{y_{t}+\bar{Y}_{t}}\right](1-\beta) A a_{t}^{\beta}=1
\end{gathered}
$$

Les firmes étant toutes identiques, nous en déduisons les conditions du premier ordre de l'équilibre symétrique de Cournot (en considérant que $n_{t} \geq 2$ firmes sont actives) :

$$
\begin{gathered}
{\left[1-\frac{1}{n_{t}}\right] \beta A a_{t}^{\beta-1}=\frac{q_{t}}{p_{t}}} \\
{\left[1-\frac{1}{n_{t}}\right](1-\beta) A a_{t}^{\beta}=\frac{1}{p_{t}}}
\end{gathered}
$$

Par ailleurs, nous supposons que l'entrée et la sortie des firmes de l'économie se font sans coût. Une condition de profits nuls détermine le nombre $n_{t}$ d'entre elles entrées à l'équilibre:

$$
p_{t} A\left(a_{t}^{\beta} l_{t}-f\right)-q_{t} k_{t-1}-l_{t}=0
$$

En substituant les équations (15) et (16) dans cette égalité, nous obtenons $^{5}$ :

$$
n_{t}=\frac{a_{t}^{\beta} l_{t}}{f}, \text { avec } n_{t} \geq 2
$$

Cette dernière équation montre que l'entrée des firmes est pro-cyclique. Elle implique également que le taux de marge, qui est égal à $\frac{1}{n_{t}-1}$, est

5. La condition du deuxième ordre de la maximisation des proffts est satisfaite car la recette marginale est décroissante et le coût marginal constant.

6 . Nous considérons, évidemment abusivement, le nombre de ffrmes $n_{t}$ comme une variable continue. 
contra-cyclique. En effet dans ce modèle, les fluctuations du taux de marge résultent uniquement des mouvements d'entrée et de sortie des firmes, puisque l'élasticité de la demande par rapport au prix est constante.

Par ailleurs, remarquons que $f$ mesure le niveau des rendements d'échelle. Il influence également le nombre de producteurs $n_{t}$. Lorsque $f$ tend vers 0 , un nombre infini de firmes entrent et le pouvoir de marché disparaît. A la limite, les rendements deviennent constants et la concurrence parfaite. Le cas opposé est celui du duopole $\left(n_{t}=2\right)$ pour lequel le taux de marge est maximum.

\subsection{Equilibre Intertemporel}

A l'équilibre, $L_{t}=n_{t} l_{t}$. L'équation (18) peut alors se réécrire:

$$
n_{t}=\sqrt{\frac{a_{t}^{\beta} L_{t}}{f}}, \text { avec } n_{t} \geq 2
$$

Le stock de capital est prédéterminé par l'épargne des jeunes de la période précédente. Autrement dit, $K_{t-1}=a_{t} L_{t}=n_{t} k_{t-1}$. En substituant cette expression ainsi que les équations (15), (16) et (19) dans (6) et (8), nous obtenons :

$$
\begin{gathered}
K_{t}=(1-\alpha)\left[1-f^{1 / 2} a_{t}^{\frac{1-\beta}{2}} K_{t-1}^{-1 / 2}\right](1-\beta) A a_{t}^{\beta-1} K_{t-1} \\
\alpha^{\alpha}(1-\alpha)^{1-\alpha} \beta^{1-\alpha}(1-\beta) A^{2-\alpha}\left[1-f^{1 / 2} a_{t+1}^{\frac{1-\beta}{2}} K_{t}^{-1 / 2}\right]^{1-\alpha} \\
a_{t+1}^{-(1-\beta)(1-\alpha)}\left[1-f^{1 / 2} a_{t}^{\frac{1-\beta}{2}} K_{t-1}^{-1 / 2}\right] a_{t}^{\beta}=v
\end{gathered}
$$

Définition 1 Un équilibre intertemporel avec prévisions parfaites est une suite $\left(K_{t-1}, a_{t}\right) \in \mathbb{R}_{++}^{2}, t=1,2, \ldots, \infty$, telle que les relations (20) et (21) sont satisfaites, pour tout $t$.

Les équations (20) et (21) déterminent entièrement la dynamique du modèle. En effet, étant donné $\left(K_{t-1}, a_{t}\right)$, on peut déterminer de manière unique $\left(K_{t}, a_{t+1}\right)$. Les équations $(20)$ et $(21)$ définissent donc un système dynamique de dimension deux avec une variable prédéterminée, le capital. 


\section{Dynamique Locale}

Dans cette section, nous allons étudier l'émergence de fluctuations endogènes stochastiques et déterministes, c'est-à-dire l'indétermination de l'équilibre stationnaire et l'apparition de bifurcations locales. Ces fluctuations vont être caractérisées par les deux propriétés que nous avons mentionnées précédemment, à savoir la pro-cyclicité du nombre de firmes et la contra-cyclicité du taux de marge. Nous allons montrer que des cycles endogènes peuvent émerger lorsque le taux de marge est positif et variable et les rendements croissants, même si ceux-ci sont faibles. Par contre, dans le cas limite où la concurrence est parfaite et le taux de marge nul, des cycles endogènes déterministes ne peuvent pas apparaître.

Pour montrer ces résultats, nous allons utiliser la méthode géométrique développée par Grandmont, Pintus, et de Vilder (1998), mais auparavant, nous allons étudier l'existence et l'unicité de l'équilibre stationnaire.

Un équilibre stationnaire $(K, a)$ est une solution du système (20)-(21), telle que :

$$
\begin{gathered}
A\left[1-f^{1 / 2} a^{\frac{1-\beta}{2}} K^{-1 / 2}\right]=\frac{a^{1-\beta}}{(1-\alpha)(1-\beta)} \\
\frac{\alpha^{\alpha} \beta^{1-\alpha}}{(1-\alpha)(1-\beta)^{1-\alpha}} a=v
\end{gathered}
$$

Nous constatons qu'il existe une unique solution a à l'équation (23) et, étant donné $a$, il existe une unique solution $K$ à l'équation (22). Il existe donc un unique équilibre stationnaire. En utilisant la méthode de Cazzavillan, Lloyd-Braga, et Pintus (1998), nous établissons l'existence d'un équilibre stationnaire normalisé $(K, a)=(1,1)$ en choisissant de manière appropriée les paramètres $A>0$ et $v>0$, c'est-à-dire tels que:

$$
\begin{gathered}
A=\frac{1}{(1-\alpha)(1-\beta)\left(1-f^{1 / 2}\right)} \\
v=\frac{\alpha^{\alpha} \beta^{1-\alpha}}{(1-\alpha)(1-\beta)^{1-\alpha}}
\end{gathered}
$$

Comme $\alpha \in(0,1), \beta \in(0,1)$ et $f \in(0,1 / 4)$, il existe une solution unique $A, v>0$ au système $(24)-(25)$ et $(K, a)=(1,1)$ est l'unique équilibre stationnaire du système dynamique $(20)-(21){ }^{6}$

7. L'importance de l'hypothèse $f<\frac{1}{4}$ devient maintenant claire. En effet, à l'état stationnaire $(K, a)=(1,1)$, l'équation (19) s'écrit $n=f^{-(1 / 2)}$. Comme le nombre de producteurs est supérieur à deux, le coût ffxe $f$ doit être inférieur à $\frac{1}{4}$. L'inégalité stricte découle 
Proposition 1 Soient $A>0$ et $v>0$ l'unique solution du système (24)(25). Alors, l'équilibre stationnaire $(K, a)=(1,1)$ existe et est unique.

Nous pouvons maintenant appliquer la méthode développée par Grandmont, Pintus, et de Vilder (1998). Celle-ci s'applique aux systèmes dynamiques discrets non-linéaires de dimension deux et permet d'étudier la stabilité locale (l'indétermination locale) de l'équilibre stationnaire et l'émergence de bifurcations locales. Nous allons commencer par différentier le système dynamique (20)-(21) dans le voisinage de l'équilibre stationnaire $(K, a)=(1,1) .{ }^{7}$ Nous obtenons :

$$
\begin{gathered}
\frac{d K_{t}}{K}=\frac{2-f^{1 / 2}}{2\left(1-f^{1 / 2}\right)} \frac{d K_{t-1}}{K}-\frac{(1-\beta)\left(2-f^{1 / 2}\right)}{2\left(1-f^{1 / 2}\right)} \frac{d a_{t}}{a} \\
\frac{d a_{t+1}}{a}=\left[\frac{f^{1 / 2}}{(1-\alpha)(1-\beta)\left(2-f^{1 / 2}\right)}+\frac{f^{1 / 2}}{2(1-\beta)\left(1-f^{1 / 2}\right)}\right] \frac{d K_{t-1}}{K} \\
+\left[\frac{f^{1 / 2}}{(1-\alpha)(1-\beta)}-\frac{f^{1 / 2}}{(1-\alpha)(1-\beta)\left(2-f^{1 / 2}\right)}-\frac{d a_{t}}{2\left(1-f^{1 / 2}\right)}\right] \frac{\beta}{a}
\end{gathered}
$$

La trace $T$ et le déterminant $D$ de la matrice Jacobienne ont les expressions suivantes :

$$
\begin{gathered}
T=\frac{\alpha}{1-\alpha}\left(T_{1}-1\right)+T_{1} \text {, avec } T_{1}=1+\frac{\beta}{1-\beta}-\frac{f^{1 / 2}}{(1-\beta)\left(2-f^{1 / 2}\right)} \\
D=\left(\frac{\alpha}{1-\alpha}+1\right) D_{1}, \text { avec } D_{1}=\frac{\beta}{1-\beta}
\end{gathered}
$$

Comme le suggèrent Grandmont, Pintus, et de Vilder (1998), l'étude de la stabilité locale de l'équilibre stationnaire et de l'émergence de bifurcations locales peut être réalisée en se plaçant dans le plan $(T, D)$ (voir Figure 1). Une valeur propre de la matrice Jacobienne est égale à 1 lorsque $1-T+D=0$ (droite $(A C)$ ). Un valeur propre est égale à -1 lorsque $1+T+D=0$ (droite $(A B))$. Les deux valeurs propres sont complexes et conjuguées et ont un

du fait que comme nous nous intéressons à l'émergence de fiuctuations endogènes dans le voisinage de l'équilibre stationnaire, $n$ doit être strictement supérieur à 2 à l'équilibre stationnaire. Notons par ailleurs qu'à l'état stationnaire $(K, a)=(1,1), L=1<L^{*}$.

8. D'après le théorème de Hartman-Grobman, le système dynamique linéarisé a les mêmes propriétés dynamiques qualitatives que le système dynamique initial si la matrice Jacobienne est inversible et si ses valeurs propres ne sont pas de module unitaire. Voir par exemple Grandmont (1988). 
module égal à $1(D=1$ et $-2<T<2)$ lorsqu'on se trouve sur le segment $[B C]$. Par conséquent, l'équilibre stationnaire est localement stable lorsque $1-T+D>0,1+T+D>0$ et $D<1$ (à l'intérieur du triangle $(A B C)$ ), c'est un point-selle lorsque $1-T+D<0(>0)$ et $1+T+D>0(<0)$, et dans tous les autres cas, il est instable.

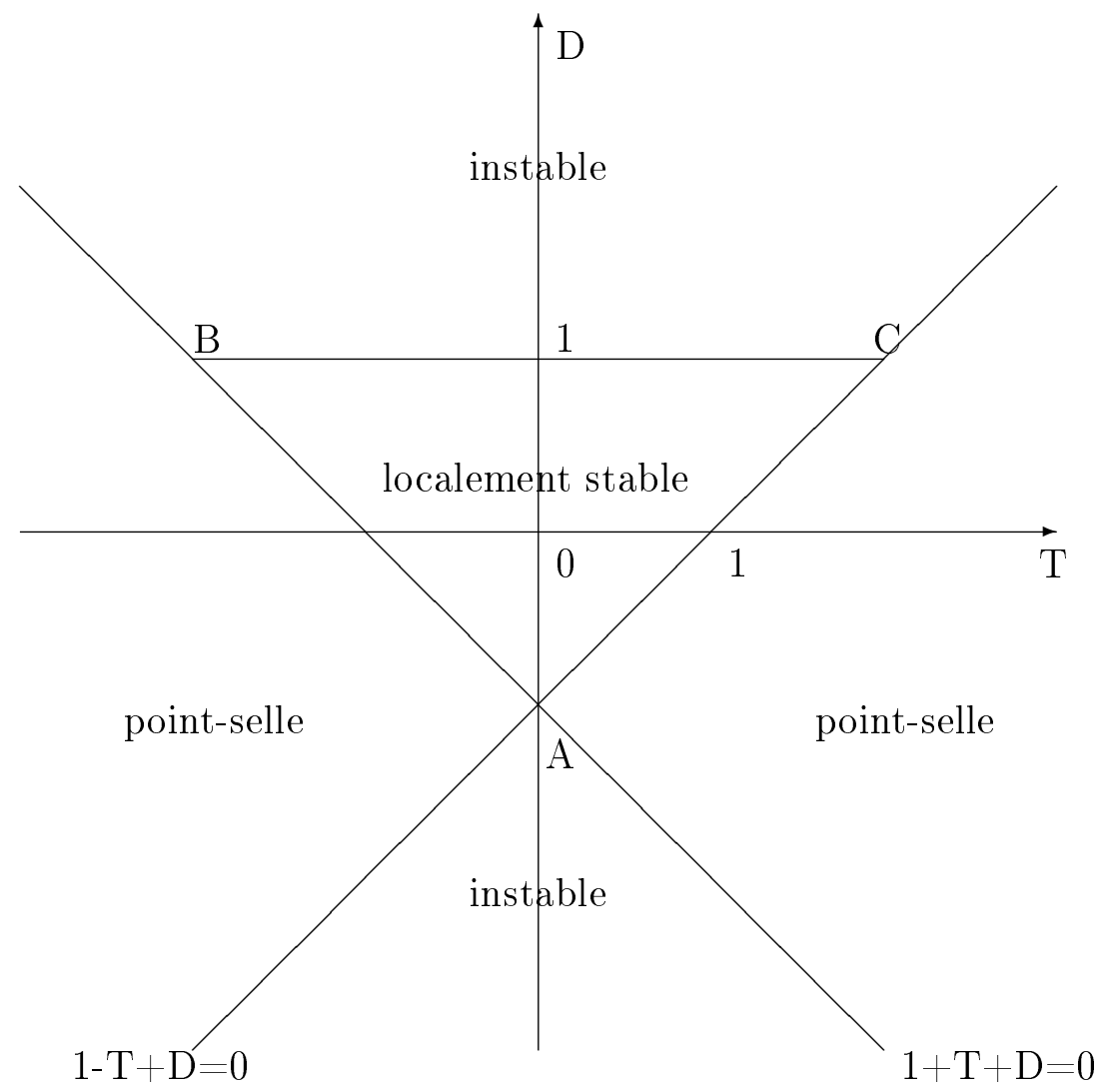

FIG. 1 - La Méthode Géométrique

Comme nous l'avons mentionné, cette méthode permet également d'étudier l'émergence de bifurcations locales. Suite au changement de valeur d'un paramètre (paramètre de bifurcation), si $(T, D)$ traverse la droite $(A B)$, une bifurcation flip apparaît, c'est-à-dire un cycle de période deux dans le voisinage de l'équilibre stationnaire. Lorsque $(T, D)$ traverse le segment $[B C]$, une bifurcation Hopf apparaît, c'est-à-dire une courbe fermée invariante dans le voisinage de l'état stationnaire.

Dans notre modèle dynamique à deux dimensions, le capital est prédéterminé par l'épargne des jeunes de la période précédente. Cela signifie que des fluctuations endogènes stochastiques apparaissent dans le voisinage de l'équilibre stationnaire lorsqu'il est localement stable (localement indéter- 
miné). Par ailleurs, des fluctuations endogènes stochastiques émergent dans le voisinage du cycle (bifurcation flip) s'il est localement stable ou dans le voisinage de la courbe fermée invariante (bifurcation Hopf) si elle est localement stable, c'est-à-dire si la bifurcation est supercritique. ${ }^{8}$

Nous allons maintenant appliquer cette méthode à notre économie et analyser ainsi l'émergence de fluctuations endogènes. Nous choisissons le facteur de la préférence pour le présent $\frac{\alpha}{1-\alpha}$ comme paramètre de bifurcation. Il varie de 0 à $+\infty$, car $\alpha \in(0,1)$. D'après les expressions (28) et (29), lorsque $\frac{\alpha}{1-\alpha}$ décrit $(0,+\infty),(T, D)$ décrit une demi-droite $\Delta$. Elle a pour extrémité $\left(T_{1}, D_{1}\right)$ lorsque $\frac{\alpha}{1-\alpha}$ est proche de 0 et sa pente $S$ est égale à :

$$
S=\frac{D_{1}}{T_{1}-1}=\frac{\beta\left(2-f^{1 / 2}\right)}{2 \beta-(1+\beta) f^{1 / 2}}
$$

Etudier la stabilité locale de l'équilibre stationnaire et l'apparition de bifurcations locales revient donc à analyser la demi-droite $\Delta$, autrement dit la position de $\left(T_{1}, D_{1}\right)$ et la valeur de la pente $S$.

Nous allons faire l'hypothèse suivante concernant le paramètre $\beta$ :

Hypothèse $1 \beta<\frac{1}{2}$.

Cette hypothèse qui restreint les valeurs du paramètre $\beta$ signifie simplement que la part du revenu du capital dans la production ${ }^{00}$ est inférieure à $\frac{1}{2}$. En outre, cette hypothèse n'est pas contredite par les résultats empiriques.

Nous allons maintenant étudier la position de $\left(T_{1}, D_{1}\right)$ dans le plan $(T, D)$. D'après les expressions (28) et (29), nous remarquons que $T_{1}>0$ et $D_{1}>0$. Par ailleurs,

$$
1-T_{1}+D_{1}=\frac{f^{1 / 2}}{(1-\beta)\left(2-f^{1 / 2}\right)}>0
$$

De plus, d'après l'Hypothèse $1, D_{1}=\frac{\beta}{1-\beta}<1$. Cela signifie que lorsque la préférence pour le présent est très faible $\left(\frac{\alpha}{1-\alpha}\right.$ proche de 0$)$, l'équilibre stationnaire est localement stable (indéterminé) et le point $\left(T_{1}, D_{1}\right)$ se situe toujours à l'intérieur du triangle $(A B C)$ (voir Figure 2).

9. Pour plus de détails à ce sujet, consulter Guesnerie et Woodford (1992) et aussi Grandmont, Pintus, et de Vilder (1998).

10. En effet, en substituant l'équation(18) dans (15), nous obtenons $r_{t} k_{t-1}=\beta y_{t}$. Par conséquent, $\beta$ est la part du capital dans le revenu. 
La pente $S$ est supérieure à 1 si $0<f<\frac{4 \beta^{2}}{(1+\beta)^{2}} .{ }^{00}$ Quand $f>\frac{4 \beta^{2}}{(1+\beta)^{2}}$, la pente $S$ est négative. En outre, $S \in(-\infty,-1)$ si $\frac{4 \beta^{2}}{(1+\beta)^{2}}<f<\frac{16 \beta^{2}}{(1+2 \beta)^{2}}$ et la demi-droite $\Delta$ passe par le point B si $f=\frac{64 \beta^{2}}{(1+4 \beta)^{2}}$.

Nous en déduisons que si $0<f<\frac{16 \beta^{2}}{(1+2 \beta)^{2}}$, la demi-droite $\Delta$ traverse le segment $[B C]$. Quand $\frac{16 \beta^{2}}{(1+2 \beta)^{2}}<f<\frac{64 \beta^{2}}{(1+4 \beta)^{2}}$, la demi-droite $\Delta$ traverse le segment $[B C]$ puis la droite $(A B)$. Enfin, si $f>\frac{64 \beta^{2}}{(1+4 \beta)^{2}}$, la demi-droite $\Delta$ traverse uniquement la droite $(A B)$, entre le point $\mathrm{A}$ et le point $\mathrm{B}$ (voir Figure 2).

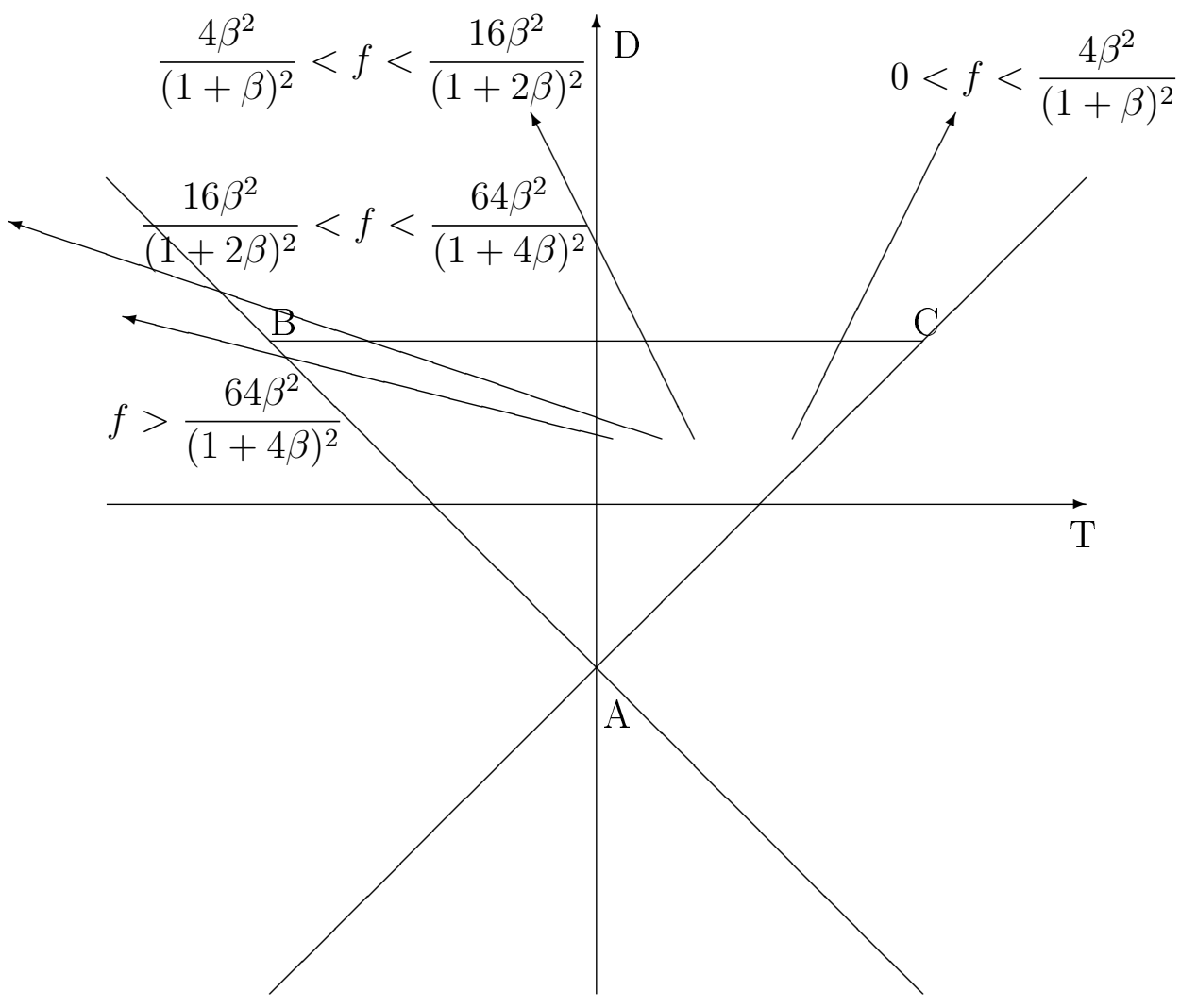

FIG. 2 - Stabilité Locale et Bifurcations

11. Dans ce cas, $T_{1}>1$ et nous pouvons déduire que $S>1$ de l'inégalité $1-T_{1}+D_{1}>0$. 
Ces constatations géométriques nous permettent d'établir les résultats suivants :

Proposition 2 Supposons que $\beta<\frac{1}{2}$ (Hypothèse 1). Alors,

1. si $0<f<\frac{16 \beta^{2}}{(1+2 \beta)^{2}}$, l'équilibre stationnaire est localement stable lorsque $\frac{\alpha}{1-\alpha}<\left(\frac{\alpha}{1-\alpha}\right)_{H}$, une bifurcation Hopf émerge lorsque $\frac{\alpha}{1-\alpha}=$ $\left(\frac{\alpha}{1-\alpha}\right)_{H}$ et l'équilibre stationnaire est instable lorsque $\frac{\alpha}{1-\alpha}>\left(\frac{\alpha}{1-\alpha}\right)_{H}$;

2. si $\frac{16 \beta^{2}}{(1+2 \beta)^{2}}<f<\frac{64 \beta^{2}}{(1+4 \beta)^{2}}$, l'équilibre stationnaire est localement stable lorsque $\frac{\alpha}{1-\alpha}<\left(\frac{\alpha}{1-\alpha}\right)_{H}$, une bifurcation Hopf émerge lorsque $\frac{\alpha}{1-\alpha}=\left(\frac{\alpha}{1-\alpha}\right)_{H}$, l'équilibre stationnaire est instable lorsque $\left(\frac{\alpha}{1-\alpha}\right)_{H}$ $<\frac{\alpha}{1-\alpha}<\left(\frac{\alpha}{1-\alpha}\right)_{F}$, une bifurcation fiip émerge lorsque $\frac{\alpha}{1-\alpha}=$ $\left(\frac{\alpha}{1-\alpha}\right)_{F}$ et l'équilibre stationnaire est un point-selle lorsque $\frac{\alpha}{1-\alpha}>$ $\left(\frac{\alpha}{1-\alpha}\right)_{F}$

3. si $f>\frac{64 \beta^{2}}{(1+4 \beta)^{2}}$, l'équilibre stationnaire est localement stable lorsque $\frac{\alpha}{1-\alpha}<\left(\frac{\alpha}{1-\alpha}\right)_{F}$, une bifurcation fiip émerge lorsque $\frac{\alpha}{1-\alpha}=\left(\frac{\alpha}{1-\alpha}\right)_{F}$ et l'équilibre stationnaire est un point-selle lorsque $\frac{\alpha}{1-\alpha}>\left(\frac{\alpha}{1-\alpha}\right)_{F}$;

$\operatorname{avec}\left(\frac{\alpha}{1-\alpha}\right)_{H}=\frac{1-2 \beta}{\beta}$ et $\left(\frac{\alpha}{1-\alpha}\right)_{F}=\frac{4-3 f^{1 / 2}}{(1+2 \beta) f^{1 / 2}-4 \beta}$.

Cette proposition montre que des fluctuations endogènes stochastiques et déterministes peuvent émerger dans notre modèle. En effet, lorsque la préférence pour le présent est suffisamment faible, l'équilibre stationnaire est localement indéterminé. Dans ce cas, des fluctuations endogènes stochastiques apparaissent dans le voisinage de l'équilibre stationnaire. De plus, des fluctuations endogènes déterministes peuvent émerger à travers l'apparition de bifurcations flip et Hopf. Des fluctuations endogènes stochastiques apparaissent alors dans le voisinage du cycle (bifurcation flip) s'il est localement 
stable ou dans le voisinage de la courbe fermée invariante (bifurcation Hopf) si elle est localement stable.

L'existence de fluctuations endogènes peut s'interpréter de la manière suivante. Supposons que l'on dévie de l'équilibre stationnaire par une baisse du coût marginal. Cela entraîne l'existence de profits positifs. De nouvelles firmes entrent et le taux de marge diminue. Le coût marginal devenant trop important par rapport au prix, certaines firmes vont sortir de l'économie, ce qui va accroître le taux de marge. Un nouveau retournement du cycle peut s'opérer ensuite avec l'entrée de nouvelles firmes.

Le coût fixe $f \in(0,1 / 4)$ est un indice du niveau du taux de marge. Le taux de marge est en effet positif et variable si $f$ est positif. Or, la Proposition 2 montre que des fluctuations endogènes déterministes peuvent émerger si le coût fixe $f$ est strictement positif. Elle montre donc que des fluctuations endogènes déterministes peuvent apparaître quelque soit la valeur du taux de marge, dès lors qu'il est positif. Par conséquent, l'émergence de cycles endogènes ne requiert pas des rendements fortement croissants, comme c'est souvent le cas dans les modèles à un secteur. Ce résultat semble ainsi en accord avec les résultats empiriques récents (Basu et Fernald (1995), Basu et Fernald (1997), Burnside (1996)). De plus, l'existence de cycles endogènes n'impose pas à la demande de travail d'être croissante. En effet, en utilisant les équations (16) et (19), nous déterminons l'élasticité de la demande de travail à l'état stationnaire $(K, a)=(1,1)$ :

$$
\frac{d(1 / p)}{d L} \frac{L}{1 / p}=\frac{1-\beta}{2} \frac{f^{1 / 2}}{1-f^{1 / 2}}-\beta
$$

D'après cette équation, la demande de travail est croissante si $f>\frac{4 \beta^{2}}{(1+\beta)^{2}}$. Or, la Proposition 2 montre que des cycles endogènes peuvent également émerger si $0<f<\frac{4 \beta^{2}}{(1+\beta)^{2}}$, c'est-à-dire si la demande de travail est décroissante. ${ }^{01}$

Lorsque $f$ tend vers 0 , un nombre infini de firmes entrent et le taux de marge disparaît. Il s'agit du cas limite où la concurrence est parfaite et les rendements constants. Alors, des cycles endogènes déterministes ne peuvent plus apparaître (bifurcations flip et Hopf). En effet, $1-T_{1}+D_{1}=0$ et la pente $S$ de la demi-droite $\Delta$ est égale à 1 . Cela signifie que $1-T+D=0$ et qu'une valeur propre est égale à 1 . Dans une telle situation, la seconde valeur propre est positive, inférieure à 1 si $\frac{\alpha}{1-\alpha}<\frac{1-2 \beta}{\beta}$ et supérieure à 1 dans le cas

12. Ce résultat est également obtenu par Coimbra (1999). 
contraire, d'après l'équation (29). Des fluctuations endogènes stochastiques apparaissent dans le voisinage de l'équilibre stationnaire si $\frac{\alpha}{1-\alpha}<\frac{1-2 \beta}{\beta}$ et l'équilibre est localement déterminé si $\frac{\alpha}{1-\alpha}>\frac{1-2 \beta}{\beta}$. Des fluctuations endogènes déterministes ne peuvent pas émerger. La concurrence imparfaite et la variabilité du taux de marge favorisent donc l'émergence de fluctuations endogènes.

\section{Conclusion}

Dans cet article, nous étudions l'émergence de fluctuations endogènes dans un modèle à générations imbriquées, caractérisé par un taux de marge variable et des rendements croissants. La variabilité du taux de marge est due au nombre endogène et variable de producteurs. Nous utilisons un modèle de Cournot avec libre entrée dans lequel les rendements croissants se traduisent par l'existence d'un coût fixe. Le nombre de firmes est pro-cyclique et le taux de marge contra-cyclique. Nous considérons par ailleurs le cas particulier où l'offre de travail est infiniment élastique.

Nous montrons que l'équilibre stationnaire peut être localement indéterminé et des cycles endogènes peuvent émerger lorsque le taux de marge est positif et variable, c'est-à-dire lorsque les rendements sont croissants, aussi faiblement que ce soit. Par contre, dans le cas limite où la concurrence est parfaite et le taux de marge disparaît, l'équilibre stationnaire peut être localement indéterminé, mais des fluctuations endogènes déterministes ne peuvent pas apparaître. La concurrence imparfaite et la variabilité du taux de marge ont donc un rôle primordial sur l'émergence de fluctuations endogènes. L'émergence de cycles endogènes déterministes requiert l'existence de rendements croissants, mais ils n'ont pas besoin d'être importants. En outre, ces cycles endogènes peuvent émerger si la demande de travail n'est pas croissante.

\section{Références}

Basu, S., et J. Fernald (1995): “Are Apparent Productive Spillovers a Figment of Specification Error?," Journal of Monetary Economics, 36, $165-188$.

(1997): "Returns to Scale in US Production: Estimates and Implications," Journal of Political Economy, 105, 249-283. 
BenhabiB, J., et R. H. DAY (1982): "A Characterization of Erratic Dynamics in the Overlapping Generations Model," Journal of Economic Dynamics and Control, 4, 37-55.

Benhabib, J., et R. E. A. FARmer (1994): "Indeterminacy and Increasing Returns," Journal of Economic Theory, 63, 19-41.

Bils, M. (1987): "The Cyclical Behavior of Marginal Cost and Price," American Economic Review, 77, 838-855.

Boldrin, M., ET M. WoOdFord (1990): "Equilibrium Models Displaying Endogenous Fluctuations and Chaos," Journal of Monetary Economics, 25, 189-222.

Burnside, C. (1996): "Production Function Regressions, Returns to Scale, and Externalities," Journal of Monetary Economics, 37, 177-201.

Caballero, R. J., et R. K. Lyons (1992): "External Effects in US Procyclical Productivity," Journal of Monetary Economics, 29, 209-225.

Cazzavillan, G. (2001): "Indeterminacy and Endogenous Fluctuations with Arbitrarily Small Externalities," forthcoming in Journal of Economic Theory.

Cazzavillan, G., T. Lloyd-Braga, et P. Pintus (1998): "Multiple Steady States and Endogenous Fluctuations with Increasing Returns to Scale in Production," Journal of Economic Theory, 80, 60-107.

ColmbrA, R. (1999): "Efficiency Wages, Increasing Returns and Endogenous Fluctuations," Discussion Paper 99/06, University of York.

Coimbra, R., T. Lloyd-Braga, et L. Modesto (2000): "Unions, Increasing Returns and Endogenous Fluctuations," IZA Discussion Paper 229, Bonn.

Cook, D. (2001): "Time to Enter and Business Cycles," Journal of Economic Dynamics and Control, 25, 1241-1261.

D'Aspremont, C., R. Dos Santos Ferreira, et L.-A. Gérard-Varet (1995): "Market Power, Coordination Failures and Endogenous Fluctuations," dans The New Macroeconomics: Imperfect Markets and Policy Effectiveness, ed. par H. D. Dixon, et N. Rankin, pp. 94-138, Cambridge. Cambridge University Press.

DE Vilder, R. G. (1996): "Complicated Endogenous Business Cycles under Gross Substituability," Journal of Economic Theory, 69, 416-442.

Dos Santos Ferreira, R., et T. Lloyd-Braga (2000): "Nonlinear Endogenous Fluctuations with Free Entry and Variable Markups," miméo, Strasbourg.

Gali, J. (1994): "Monopolistic Competition, Business Cycles, and the Composition of Aggregate Demand," Journal of Economic Theory, 63, 73-96. 
Grandmont, J.-M. (1985): "On Endogenous Competitive Business Cycles," Econometrica, 53, 995-1045.

(1988): "Nonlinear Difference Equations, Bifurcation and Chaos: an Introduction," Working Paper 8811, CEPREMAP.

Grandmont, J.-M., P. Pintus, et R. De Vilder (1998): "Capital-labour Substitution and Competitive Nonlinear Endogenous Business Cycles," Journal of Economic Theory, 80, 14-59.

Guesnerie, R., Et M. Woodford (1992): "Endogenous Fluctuations," dans Advances in Economic Theory: Proceedings of the $6^{\text {th }}$ World Congress of the Econometric Society, ed. par J.-J. Laffont, vol. 2, pp. 289-412, Cambridge, Ma. Cambridge University Press.

Hansen, G. (1985): "Indivisible Labor and the Business Cycle," Journal of Monetary Economics, 16, 309-325.

Jacobsen, H. J. (1998): "Endogenous Product Diversity and Endogenous Business Cycles," Discussion Paper 98-15, University of Copenhagen.

Kuhry, Y. (2001): "Endogenous Fluctuations in a Cournotian Monopolistic Competition Model with Free Entry and Market Power Variability," Research in Economics, 55, 389-412.

Martins, J. O., et S. Scarpetta (1999): "The Levels and Cyclical Behaviour of Mark-ups across Countries and Market Structures," Economics Department Working Papers $n^{\circ} 213$ OCDE.

Portier, F. (1995): "Business Formation and Cyclical Markups in the French Business Cycle," Annales d'Economie et de Statistique, 37/38, 411440.

Reichlin, P. (1986): "Equilibrium Cycles in an Overlapping Generations Economy with Production," Journal of Economic Theory, 40, 89-103.

RivarD, B. (1994): "Monopolistic Competition, Increasing Returns, and Selffulfilling Prophecies," Journal of Economic Theory, 62, 346-362.

Rotemberg, J. J., et M. Woodford (1991): "Markups and the Business Cycle," dans NBER Macroeconomics Annual, ed. par O. J. Blanchard, et S. Fischer, pp. 63-128, Cambridge, Ma. MIT Press.

Seegmuller, T. (2001): "Capital-labour Substitution and Endogenous Fluctuations: a Monopolistic Competition Approach with Variable Markup," miméo, Strasbourg.

Weder, M. (2000): "Animal Spirits, Technology Shocks and the Business Cycle," Journal of Economic Dynamics and Control, 24, 273-295.

WoOdFord, M. (1986): "Stationary Sunspot Equilibria in a Finance Constrained Economy," Journal of Economic Theory, 40, 128-137. 
_ (1991): "Self-fulfilling Expectations and Fluctuations in Aggregate Demand," dans New Keynesian Economics, ed. par N. G. Mankiw, et D. Romer, vol. 2, pp. 77-110, Cambridge. MIT Press. 